American Journal of Immunology 1 (1): 55-59, 2005

ISSN 1553-619X

(C) 2005 Science Publications

\title{
Heparin-PF4 Antibodies in Heparin Induced Thrombocytopenia: Its Relationship with FcgRIIa Polymorphism
}

\author{
${ }^{1}$ Meganathan Kannan, ${ }^{1}$ Firdos Ahmad, ${ }^{2,3}$ Sarfraz Ahmad, ${ }^{1}$ Shailaja Kale ${ }^{2}$ Jawed Fareed and ${ }^{1}$ Renu Saxena \\ ${ }^{1}$ Department of Hematology, All India Institute of Medical Sciences, Ansari Nagar \\ New Delhi 100 029, India \\ ${ }^{2}$ Loyola University of Chicago, Stritch School of Medicine, Maywood, IL 60153, USA \\ ${ }^{3}$ Florida Hospital Cancer Institute, Orlando, FL 32804, USA
}

\begin{abstract}
We did the pilot study, in an attempt to determine the prevalence of HIT in Indians and determine its relationship, if any, to FcyRIIa polymorphism, the occurrence of heparin-induced thrombocytopenia (HIT) was investigated in 33 Indian patients undergoing cardiovascular surgery (CVS) who received unfractionated heparin (UFH). Platelet counts were performed prior to the initiation of UFH therapy and 5-16 days, post-therapy. The fall in patients' platelet count $>35 \%$ of the baseline value or $<100,000 \mu \mathrm{L}^{-1}$ was considered to be suggestive of HIT syndrome. Heparin-induced platelet aggregation (HIPA), Enzyme Linked Immunosorbant assay (ELISA) and Serotonin Release Assay (SRA) tests were performed in all the patients to detect the antibodies formed against the heparin and platelet factor 4 complex (H-PF4). DNA analysis for Fc $\gamma$ RIIa polymorphism was done by allele specific PCR. Thrombocytopenia was found to be present in $10(30 \%)$ patients. Of these, 5 patients were found to be positive by HIPA, ELISA and SRA tests. These 5 patients were considered to have classic HIT syndrome. One of these patients had bleeding while the others were asymptomatic. One of the HIT positive patients was found to be homozygous for FcyRIIa ${ }^{\mathrm{His} / \mathrm{His}}$ polymorphism and others were heterozygous, had one defective allele with single amino acid change (Arg $131 \mathrm{His}$ ) at Fc $\gamma$ RIIa platelet receptor. Frequency of occurrence of homozygous $\left(\mathrm{Fc} \gamma \mathrm{RIIa}{ }^{\mathrm{His} / \mathrm{His})}\right.$ and heterozygous (FcyRIIa ${ }^{\mathrm{Arg} / \mathrm{His}}$ ) polymorphism was higher in patients with HIT (100\%) than in the normal population (68\%). The prevalence of HIT in the pilot study (15\%) was higher than that in the west $(5 \%)$ but comparable to that from Japan (12\%) (14). However, the Occurance of FcyRIIa polymorphism in Indian $(68 \%)$ was higher than in the east $(51 \%)$ and comparable to that in the west $(78 \%)$. Thus it appears that the FcyRIIa polymorphism may have some relation to occurrence of HIT. Higher prevalence of Fc $\gamma$ RIIa polymorphism in Indian may predispose to higher prevalence of HIT in India. Thus, it is recommended that, heparin-treated patients should be routinely tested for not only aPTT but also for platelet count, HPF4 antibodies and FcrRIIa polymorphism analysis. From the pilot study, it shows that absence of FcyRIIa receptor polymorphism may suggest low or negligible risk of development of HIT antibodies. However, further studies required with more patients using the same protocol.
\end{abstract}

Key words: Heparin-induced thrombocytopenia, heparin-PF4 antibodies, laboratory methods, polymorphism, prevalence, Indian subjects

\section{INTRODUCTION}

Heparin induced thrombocytopenia (HIT) is emerging as an important complication not only of unfractionated heparins (UFH), but also of low molecular weight heparins (LMWH) which are widely used as antithrombotic agent for both therapeutic as well as surgical indications ${ }^{[1]}$. Ethnic variation in prevalence of HIT is known to exist ${ }^{[2]}$. In the west, HIT has been found in $2-5 \%$ of patients on UFH and $0.1-1 \%$ on $\mathrm{LMWH}^{[3,4]}$, whereas in east (Japan) it has been found in $12 \%{ }^{[4]}$. It is important to identify HIT because of its impact in management of these patients clinically. Fifty percent of HIT patients may be asymptomatic, $10 \%$ may have bleeding manifestation due to thrombocytopenia, $10 \%$ may have skin necrosis and approximately $30 \%$ of HIT patients may have thrombotic commlications due to platelet activation ${ }^{[5-7]}$. If the patients of HIT have thrombosis, heparin needs to be stopped and replaced by thrombin inhibitors. HIT occurs due to the formation of antibodies against heparin-PF4 complex (the negatively charged heparin polysaccharide molecules bind to positively charged protein tetramer, PF4 to form a complex), which cause

Corresponding Author: Dr. Renu Saxena, Professor, Department of Haematology, All India Institute of Medical Sciences, IRCH Building 1st Floor, Ansari Nagar, New Delhi-110 029, India, Tel: +91-11-2659-3642, Fax: +91-11-26588663 
platelet activation, leading to thrombosis ${ }^{[8,9]}$. Immune mediated HIT is diagnosed by $50 \%$ drop in platelet count occurring after 4-15 days of heparin therapy ${ }^{[10,11]}$. It is caused by binding of antibodies to $\mathrm{Fc} \gamma$ receptor present on platelets thereby activating them. The receptor is synthesized by FcभRIIa gene. The gene encoding the receptor is polymorphic. A point mutation (G507A) causes an amino acid change Arg-His at 131 position of Fc $\gamma$ RIIa protein ${ }^{[12]}$. The polymorphism include G507A mutation in one allele results in Arg 131 His (Heterozygous) and G507A mutation in both the alleles results in Arg 131 Arg (Homozygous). In wild type, since there is no mutation it is Arg $131 \mathrm{Arg}$. Ethnic variations in the occurrence of this polymorphism are well documented ${ }^{[13]}$. In the west the polymorphism is seen in $74 \%$ of normal population, whereas in the east, it has been found in $51 \%$ of normal population $^{[14]}$. There are postulations suggesting that prevalence of Fc $\gamma$ RIIa polymorphism may affect that of HIT, some studies show this to be correct ${ }^{[12,13]}$, whereas others negate the association of FcyRIIa polymorphism with HIT $^{[19]}$. However, some studies negate the association of Fc $\gamma$ RIIa with HIT.

Since no study on HIT in Indians is available, the present study was conducted to determine the prevalence of HIT in Indians and to look for association of FcyRIIa polymorphism with HIT.

\section{MATERIALS AND METHODS}

Study Subjects: Patients $(n=33)$ undergoing cardiovascular surgery who received UFH (Biologic E. Ltd., Hyderabad, India) were studied at the All India Institute of Medical Sciences, New Delhi. The indications for heparin therapy were coronary artery bypass graft $(\mathrm{CABG})$, cardiopulmonary bypass (CPB), aortic valve replacement (AVR) and double valve replacement (DVR). Heparin was administered intravenously at the dosage of $4 \mathrm{mg} \mathrm{kg}^{-1}$ body weight (20,000 to $30,000 \mathrm{IU})$. The duration of the treatment varied from 5-16 days. These time points were chosen based on the literature, which suggests that the optimal generation (seroconversion) of HPF4 antibody occurs after several days of heparin administration. Patients with thrombocytopenia due to other drugs, septicemia and/or disseminated intravascular coagulopathy (DIC), were excluded from the study.

Blood samples were collected in trisodium citrate (1:9) from the patients before heparin (baseline) and after 5-16 days of its administration. Platelet counts, activated partial thromboplastin time (aPTT) and prothrombin time (PT) levels were monitored and the functional and immunologic assays for the laboratory diagnosis of HIT were performed on the patients plasma utilizing the following methods.

Heparin-induced platelet aggregation (HIPA): This is a functional assay ${ }^{[15]}$. HIPA was performed by mixing the platelet-poor plasma (PPP) of patients with platelet-rich plasma (PRP) of normal individuals (pre- determined to be HIT antibody-reactive donors) in the presence of therapeutic UFH level. Prior to assay, the patient's PPP was heat-inactivated by incubating at $56^{\circ} \mathrm{C}$ for $45 \mathrm{~min}$ and a clear supernatant was obtained after centrifugation at 3,000 rpm for $15 \mathrm{~min}$. To $140 \mu \mathrm{L}$ of pre-warmed donor PRP, $220 \mu \mathrm{L}$ of patient's heatinactivated PPP was added to a cuvette containing a stir bar, which was placed in the aggregometer (ChronoLog, Havertown, PA). To this, $40 \mu \mathrm{L}$ of $2.5 \mathrm{U} \mathrm{mL}^{-1}$ of $\mathrm{UFH}$ was added (final UFH concentration $=0.25$ $\mathrm{U} \mathrm{mL}^{-1}$ ) and the aggregation response was monitored for up to $5 \mathrm{~min}$. The test was considered positive for HIT, if there was $\geq 20 \%$ platelet aggregation response due to HPF4 antibodies as per the assay criteria. For all the negative responses, the viability of platelets was checked with arachidonic acid-induced aggregation (Fig. 1).

${ }^{14}$ C-serotonin release assay (SRA): This test was performed using radioactive ${ }^{14} \mathrm{C}$-serotonin (Amersham Biosciences Corp., Piscataway, NJ) according to the method of Sheridan et al. ${ }^{[16]}$. Briefly, the normal donor PRP (obtained from HIT antibody-reactive donors) was labeled with $0.1 \mu \mathrm{Ci} \mathrm{mL} \mathrm{mL}^{-1}{ }^{14} \mathrm{C}$-serotonin for $45 \mathrm{~min}$ at $37^{\circ} \mathrm{C}$. Platelets were washed with calcium- and albumin-free Tyrode's (CAF) buffer followed by albumin-free Tyrode's (AFT) buffer. Patients' plasma samples were heat-inactivated at $56^{\circ} \mathrm{C}$ for $45 \mathrm{~min}$ to remove residual enzymatic activity. Seventy micro liters of labeled donor platelets were incubated with 20 $\mu \mathrm{L}$ of heat-treated patient plasma and $10 \mu \mathrm{L}$ saline or UFH (final concentrations: 0.1 and $100 \mathrm{U} \mathrm{mL}^{-1}$ ) for 60 min at room temperature with continuous and gentle shaking. The release reaction was stopped by the addition of $100 \mu \mathrm{L}$ of $4 \%$ EDTA solution (prepared in saline) to each well. The content was supplemented by centrifugation at $3,000 \mathrm{rpm}$ for $5 \mathrm{~min}$ and the radioactivity in the supernatant was measured by the scintillation counting. For quantification, $100 \mu \mathrm{L}$ of supernatant was added to the $5 \mathrm{~mL}$ of scintillation fluid (Universal LSC Cocktail; Sigma Chem. Co., St. Louis, MO) and the radioactivity was measured for at least 2 min. A test was considered positive for the presence of functional HPF4 antibodies, if the serotonin release was observed $\geq 20 \%$. Appropriate negative and positive controls were carried out simultaneously.

Measurement of HPF4 antibody titers (immunologic assay): To determine the presence of HPF4 antibody titers (optical density, OD) in HIT suspected patients' plasma samples, a commercially available ELISA test $^{[15]}$, obtained from the Genetic Testing Institute (GTI), Inc. (Brookfield, WI) was used. All the samples were run in duplicates and average values thus obtained are reported.

\section{Polymorphism study}

Nested SSP-PCR: DNA was extracted from peripheral blood by traditional phenol chlorophorm method. 

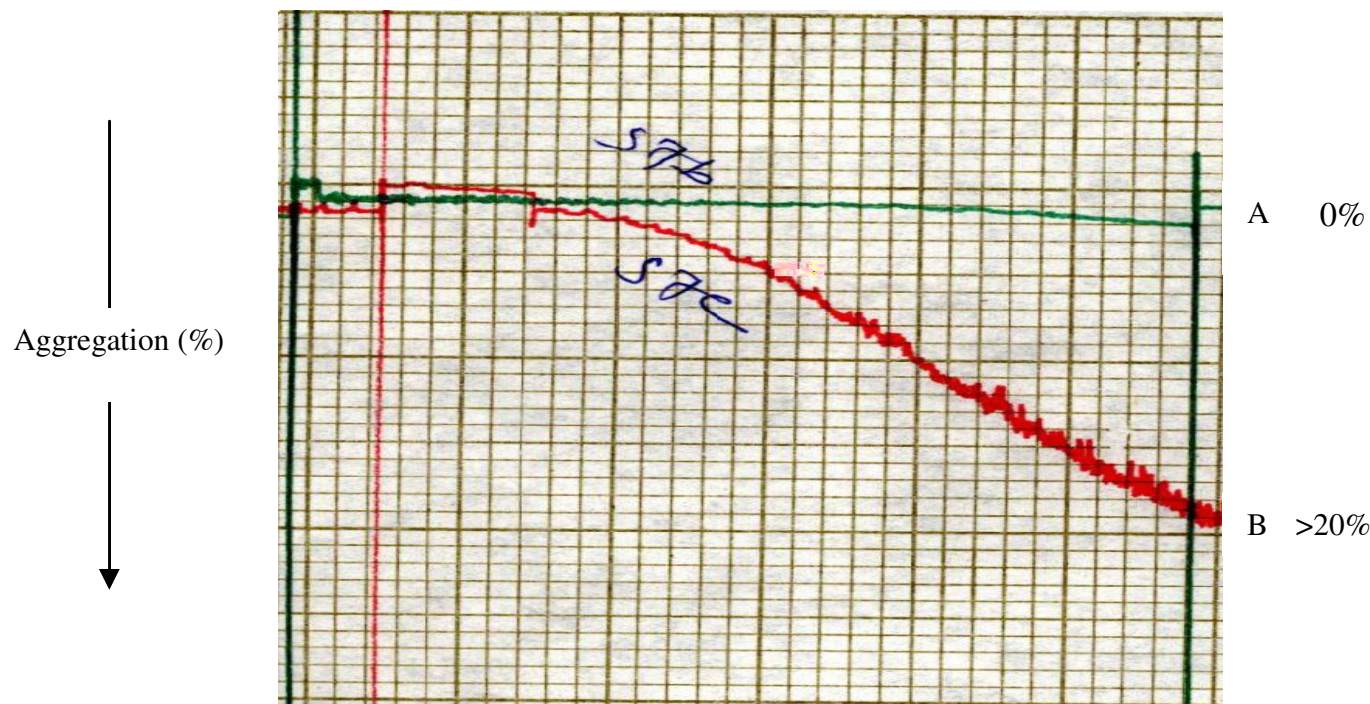

Time (in Min)

Fig. 1: Heparin Induced Platelet Aggregation chart shows absent platelet aggregation on stimulation with heparin in normal (A) and in HIT patient (B) tested on day 7 after heparin administration

Table 1: Detection of HIT-associated HPF4 antibodies by ELISA and HIPA in patients on heparin

\begin{tabular}{lllll}
\hline Patients on Heparin & Patient Number (\%) & HIPA Positivity & SRA Positivity & ELISA Positivity \\
\hline Thrombocytopenia* & $10(30 \%)$ & $2(6 \%)$ & $4(12 \%)$ & $2(6 \%)$ \\
Normal Platelet Count & $23(70 \%)$ & $0(0 \%)$ & $1(3 \%)$ & $2(6 \%)$ \\
Normal Controls & 20 & $0(0 \%)$ & $0(0 \%)$ & $0(0 \%)$ \\
\hline
\end{tabular}

*Indicates the platelet count reduced to $>35 \%$ of the initial value (baseline) or $<100,000 / \mu \mathrm{L}$

Table 2: FcgRIIa polymorphism in Indians

\begin{tabular}{lclll}
\hline & No. of Patients & FcgRIIa polymorphism & \\
& & - & \\
& & Arg/Arg & Arg/His & His/His \\
\hline HIT positive & 5 & $0(0 \%)$ & $4(80 \%)$ & $1(20 \%)$ \\
Normal Controls & 50 & $16(32 \%)$ & $33(66 \%)$ & $1(2 \%)$ \\
\hline
\end{tabular}

To genotype the Fc $\gamma$ RIIa-R-H131 polymorphism, nested sequence-specific primer-polymerase chain reaction (SSPPCR) method was done. After the first PCR amplification, using primer pair P52 (58-GAA GAG CTG CCC ATG CTG) and P63 (CAAGCC TCT GGT CAA GGT C), a 1,000-bp product obtained was reamplified using sequence-specific primers ${ }^{[17]}$. The sequence-specific sense primers $\mathrm{P} 5 \mathrm{G}$ (specific for G507) and P4A (specific for A507) are located at the polymorphic site on exon 4 and a common antisense primer, P13, is located on intron 4. A 578-bp fragment from the $\gamma$ globulin promoter region gene was used as an internal positive control (Fig. 2).

PCR conditions were as follows: 1 cycle at $95^{\circ} \mathrm{C}$ for $5 \mathrm{~min}, 55^{\circ} \mathrm{C}$ for $5 \mathrm{~min}$ and $72^{\circ} \mathrm{C}$ for $5 \mathrm{~min}$. This was followed by 35 cycles of $95^{\circ} \mathrm{C}$ for 1 minute, $55^{\circ} \mathrm{C}$ for 1 minute and $72^{\circ} \mathrm{C}$ for $2 \mathrm{~min}$, ending with an extension step at $72^{\circ} \mathrm{C}$ for $10 \mathrm{~min}$. From this reaction, $1 \mu \mathrm{L}$ was reamplified in the SSP-PCR using primers P13 (CTA GCA GCT CAC CAC TCC TC) and P5G (GAA AAT CCC AGA AAT TTT TCC G) or P4A (GAA AAT
CCCAGAAAT TTT TCC A). The nested PCR conditions were $95^{\circ} \mathrm{C}$ for 5 min followed by 30 cycles of $95^{\circ} \mathrm{C}$ for $15 \mathrm{sec}, 58^{\circ} \mathrm{C}$ for $30 \mathrm{sec}$ and $72^{\circ} \mathrm{C}$ for 30 sec, with an extension step at $72^{\circ} \mathrm{C}$ for $10 \mathrm{~min}$. The PCR products were analyzed by electrophoresis on $1.5 \%$ agarose gels stained with ethidium bromide.

\section{RESULTS}

Amongst the 33 patients who underwent heparin therapy, 22 patients were males and 11 were females and their age ranged from 11 to 72 years. Ten out of 33 patients developed thrombocytopenia with platelet counts $<35 \%$ of the initial value (Table 1 ). The aPTT level was prolonged in these patients (1.5-2.0 times the normal aPTT). Of these, 4 patients were found to be positive by SRA test. One of these also had HIPA and ELISA positivity. One more patient was detected positive by HIPA and ELISA tests. This patient had bleeding while the others were asymptomatic. 


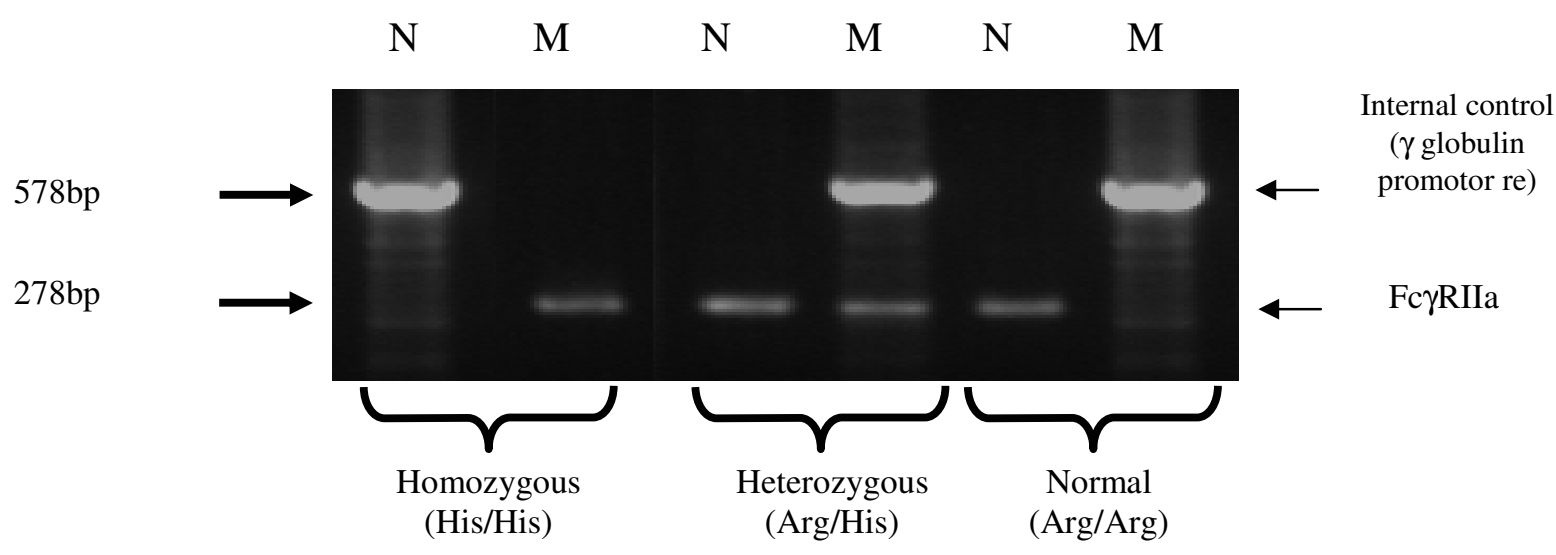

Fig. 2: Agarose gel showing presence of bands obtained using normal and mutant primers by allele specific PCR for FcrgRIIa polymorphism (Note: N:Normal primer band, M: Mutant primer band)

Since SRA and HIPA are considered to be the most sensitive/specific tests for functional HIT antibody detection,in the present study, $5(15 \%)$ patients were found to have HIT. The remaining patients were found to be negative by all the three tests, HIPA, SRAand ELISA. Amongst the 23 non-thrombocytopenic patients, HIT antibodies were not detectable by HIPA test in all, but were detectable in 2 patients by ELISA and one patient by SRA. Since they did not have any clinical symptom including thrombocytopenia, it is possible that these 3 patients had either non-functional HIT antibodies (weak affinity) or had antibodies directed against complexes formed between UFH and other chemokines of the C-X-C superfamily (such as IL-8 and/or NAP-2). All the control samples were found to be clearly negative for HPF4 antibodies as determined by all the laboratory diagnostic tests of HIT (HIPA, SRA and ELISA).

Thus, 5 patients of the 33 patients on heparin, where both HIPA and SRA were positive along with thrombocytopenia, were considered to have classic HIT syndrome. Hence the prevalence of HIT found to be $15 \%$ in Indian patients on heparin.

By the DNA analysis, one patient with thrombocytopenia was found to be homozygous for Fc $\gamma$ RIIA His/ His polymorphism and all other nine patients had at least one defective allele with single amino acid change (Arg 131 His) at Fc $\gamma$ RIIa platelet receptor (Table 2). Interestingly, the patient with homozygous polymorphism was, also detected to be positive for antibody against $\mathrm{H} / \mathrm{PF} 4$ complex by all three tests HIPA, SRA and ELISA. Though the heterozygous frequency in control population is similar to HIT patients, it has been found that $32 \%$ of normal healthy individuals carry no polymorphism in their
FcyIIa allele. In contrast, none of the patients with thromocytopenia had normal Fc $\gamma I I a$ allele.

\section{DISCUSSION}

Heparin-induced thrombocytopenia (HIT) syndrome is a complication of heparin therapy, which occurs at 5 or more days after the start of heparin therapy. It may be non-immune-mediated and immunemediated. The latter is the more severe form, also referred to as type II HIT, which might be life threatening. It is important to diagnose this clinicopathologic and potentially catastrophic syndrome because its management includes discontinuation of heparin and the administration of alternate anticoagulants (such as direct antithrombin agents). The objective of this relatively smaller clinical study was to investigate the prevalence of the symptomatic HIT patients among Indian population undergoing cardiovascular surgery while receiving UFH. Utilizing the laboratory diagnostic tests of HIT (such as platelet aggregation and SRA), 15\% of Indian patients receiving UFH anticoagulation for $\geq 5$ days developed immunemediated HIT syndrome.

The prevalence of HIT in the present study is higher than that reported earlier by Chua et al and King et al who observed the incidence of HIT as $~ 4 \%$ in east and $2-5 \%$ in the west in patients receiving $\mathrm{UFH}^{[14]}$. It is comparable to that from Japan (12\%). The sensitivity of functional assay is considered to be dependent on the heparin concentration and also on the nature of donor platelets used. In the present study, care was given to ensure that the therapeutic concentration of heparin was $0.25 \mathrm{U} \mathrm{mL}^{-1}$ and the used donor PRP was from more than one healthy individuals of ' $\mathrm{O}$ " group (pre-screened to be reactive with HIT antibodies) to include HIT sensitive platelets. 
The cause of thrombocytopenia could not be ascertained in 5 patients. It is quite likely that it may have been due to the formation of weakaffinity (non-functional) AHPF4 antibodies lead to thrombocytopenia in these patients which were not picked up by the tests. Also, it is likely that the HITassociated antibody of another type (such as the complexes formed between the heparin molecule and with other chemokines of the C-X-C super family such as IL-8 or NAP-2), may have been present. The literature also suggests that a wide variation in the prevalence, seroconversion and functionality of HITassociated antibodies exists and is largely dependent on the patient population, type/route/dosage/duration of heparin used and the diagnostic tests employed ${ }^{[18]}$.

Occurrence of polymorphism in Indians was observed to be higher than studies in the east, e.g Japan, where it is $51 \%$ but comparable to that in the west (78\%). Moreover, all cases with HIT in this study showed presence of Fc $\gamma$ RIIA polymorphism. It is postulated that the higher prevalence of Fc $\gamma$ RIIA polymorphism in Indians may contribute to higher prevalence of HIT in India.

It is concluded that HIT does occur in India, especially in patients with presence of FcyRIIa polymorphism and it is suggested that in patients on heparin, not only aPTT but also platelet count, HPF4 antibodies and FcyRIIa polymorphism analysis should be done. Absence of Fc $\gamma$ RIIa receptor polymorphism may suggest low or negligible risk of development of HIT antibodies. However, further studies required with more patients using the same protocol.

\section{REFERENCES}

1. King, D.J. and J.G. Kelton, 1984. Heparinassociated thrombocytopenia. Ann. Intern. Med., 100: 535-540.

2. Visentin, G.P., M. Malik and K.A. Cyganiak et al., 1996. Patients treated with unfractionated heparin during open heart surgery are at high risk to form antibodies reactive with heparin-platelet factor 4 complexes. J. Lab. Clin. Med., 128: 376-83.

3. Chong, B.H., $1995 . \quad$ Heparin-induced thrombocytopenia. Br. J. Haematol., 89:431-439.

4. Warkentin, T.E., M.N. Levine and J. Hirsh et al., 1995. Heparin-induced thrombocytopenia in patients treated with low-molecular-weight heparin or unfractionated heparin. N. Engl. J. Med., 332: 1330-1335.

5. Warkentin, T.E., 1999. Heparin induced thrombocytopenia. Annu. Rev. Med., 50: 129-47.

6. Warkentin, T.E. and J.G. Kelton, 1996. A 14 year study of heparin induced thrombocytopenia. Am. J. Med., 101: 502-7.

7. Nand, S., W. Wong and B. Yuen et al., 1997. Heparin Induced thrombocytopenia with thrombosis: Incidence, analysis of risk factors and clinical outcomes in 108 consecutive patients treated as a single institution. Am. J. Hematol., 56: 12-16.

8. Amiral, J., A. Marfaing-Koka, M. Poncz and D. Meyer, 1998. The biological basis of heparininduced thrombocytopenia. Platelets, 9: 77-91.

9. Amiral, J., 1999. Antigens involved in heparininduced thrombocytopenia. Semin. Hematol., 36 (Suppl.1): 7-11.

10. Brieger, D.B., K.H. Mak and K. Kottke-Marchant, 1998. Heparin-induced thrombocytopenia. J. Am. Coll. Cardiol., 31:1449-1459.

11. Fabris, F., S. Ahmad, G. Cella, W.P. Jeske, J.M. Walenga and J. Fareed, 2000. Pathophysiology of heparin-induced thrombocytopenia: Clinical and diagnostic implications-A review. Arch. Pathol. Lab. Med., 124: 1657-1666.

12. Brandt, J.T., C.E. Isenhart, J.M. Osborne, A. Ahmed and C.L. Anderson, 1995. On the role of platelet Fc gamma RIIa phenotype in heparininduced thrombocytopenia. Thromb. Haemost., 74:1564-72.

13. Lehrnbecher, T.L., C.B. Foster, S. Zhu, D. Venzon, S.M. Steinberg, K. Wyvill, J.A. Metcalf, S.S. Cohen, J. Kovacs, R. Yarchoan, A. Blauvelt and S.J. Chanock, 2000. Variant genotypes of FcgammaRIIIA influence the development of Kaposi's sarcoma in HIV-infected men. Blood, 95: 2386-90.

14. Ohno, H. and M. Yokoi, 2002. Intraoperative management for a patient with heparin-induced thrombocytopenia (HIT) undergoing off-pump coronary bypass surgery using argatroban, a direct thrombin inhibitor Masui. 51: 30-3.

15. Kannan, M., R. Ahmed, S. Kale, S. Ahmad, J. Fareed and R. Saxena, 2004. Laboratory diagnosis of heparin-induced thrombocytopenia in Asian Indians as investigated with functional and immunologic methods. Clin. Appl. Thromb. Hemost., 10: 51-4.

16. Sheridan, D., C. Carter and J.G. Kelton, 1986. A diagnostic test for heparin-induced thrombocytopenia. Blood, 67: 27-30.

17. Carlsson, L.E., S. Santoso, G. Baurichter, H. Kroll, S. Papenberg, P. Eichler, N.A. Westerdaal, V. Kiefel, J.G. van de Winkel and A. Greinacher, 1998. Heparin-induced thrombocytopenia: new insights into the impact of the FcgammaRIIa-RH131 polymorphism. Blood, 92: 1526-31.

18. Warkentin TE. 2004. Heparin-induced thrombocytopenia: diagnosis and management. Circulation.110(18):e454-8.

19. Gruel, Y., C. Pouplard, D. Lasne, C. MagdelaineBeuzelin, C. Charroing and H. Watier, 2004. The homozygous FcgammaRIIIa-158V genotype is a risk factor for heparin-induced thrombocytopenia in patients with antibodies to heparin-platelet factor 4 complexes. Blood, 104: 2791-3. 